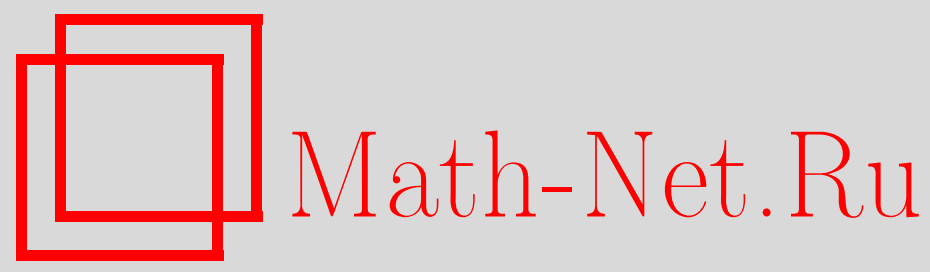

В. В. Смагин, О скорости сходимости проекционноразностных методов для гладко разрешимых параболических уравнений, Матем. заметки, 2005, том 78, выпуск 6, 907-918

DOI: https://doi.org/10.4213/mzm2662

Использование Общероссийского математического портала Math-Net.Ru подразумевает, что вы прочитали и согласны с пользовательским соглашением http://www.mathnet.ru/rus/agreement

Параметры загрузки:

IP : 52.90 .164 .192

26 апреля 2023 г., 15:39:11

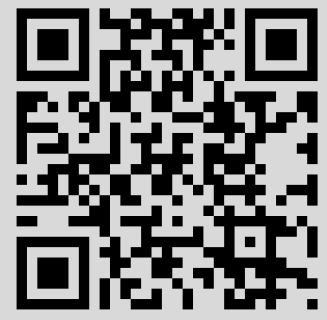




\title{
О СКОРОСТИ СХОДИМОСТИ ПРОЕКЦИОННО-РАЗНОСТНЫХ МЕТОДОВ ДЛЯ ГЛАДКО РАЗРЕШИМЫХ ПАРАБОЛИЧЕСКИХ УРАВНЕНИЙ
}

\section{В. В. Смагин}

\begin{abstract}
Линейная параболическая задача в сепарабельном гильбертовом пространстве решается приближенно проекционно-разностньм методом. Дискретизация задачи по пространству проводится методом Галёркина с ориентацией на конечномерные подпространства типа конечных элементов, а по времени используются неявная схема Эйлера и модифицированная схема Кранка-Николсон. Установлены равномерные по временной сетке и среднеквадратичные по пространству оценки погрешности приближенных решений. Эти оценки характеризуют скорость сходимости погрешностей к нулю как по временной переменной, так и по пространственным переменным.
\end{abstract}

Библиография: 10 названий.

1. Описание задачи. В работах [1] и [2] изучалась сходимость в сильных нормах проекционно-разностных методов для гладко разрешимых параболических уравнений. В [1] дискретизация задачи по времени проводится по неявной схеме Эйлера, а в [2] по модифицированной схеме Кранка-Николсон. В [3] и [4] показано соответственно, что для подпространств типа конечных элементов в более слабой среднеквадратичной норме скорость сходимости погрешностей к нулю по пространственным переменным увеличивается на порядок.

В данной работе установлено, что при оценке погрешности приближенных решений в промежуточной норме, равномерной по временной сетке и среднеквадратичной по пространству, скорость по пространственным переменным сходимости погрешностей к нулю увеличивается на полпорядка.

Для стационарных задач, решаемых проекционным методом, этот эффект достаточно хорошо известен, его обоснование назьвают леммой Обэна-Нитше (см., например, [5, c. 139]), и при этом скорость сходимости возрастает на порядок.

В случае, когда параболическая задача общего вида решается приближенно полудискретным проекционным методом (дискретизация проводится только по пространственньм переменньм), обоснование подобного факта для общего случая проекционных подпространств типа конечных элементов дано в [3] и [6]. Для весьма частного случая одномерных параболических задач и для кусочно линейных базисных функций подобный результат для проекционно-разностного метода с неявной схемой Эйлера по времени приводится в $[7$, с. 217].

Сформулируем исходную параболическую задачу в вариационной форме. Пусть $V$ и $H$ - сепарабельные гильбертовы пространства такие, что $V \subset H$ и вложение плотно и непрерывно. Через $V^{\prime}$ обозначим пространство, двойственное $V$. Отождествляя 
$H$ со своим двойственным, получим $V \subset H \subset V^{\prime}$. Под выражением вида $(z, v)$ далее понимаем значение функционала $z \in V^{\prime}$ на элементе $v \in V$. В случае $z \in H$ выражение $(z, v)$ совпадает со скалярньм произведением элементов $z, v \in H$. Норму элемента $z$ в пространстве $H$ обозначаем $\|z\|$.

Пусть для $t \in[0, T]$, где $T<\infty$, на $u, v \in V$ определено семейство полуторалинейных симметричных форм $a(t, u, v)$. Для всех $t \in[0, T]$ и $u, v \in V$ вьполнено

$$
|a(t, u, v)| \leqslant M_{1}\|u\|_{V}\|v\|_{V}, \quad a(t, u, u) \geqslant \delta\|u\|_{V}^{2}, \quad \delta>0 .
$$

Считаем, что функция $t \rightarrow a(t, u, v)$ абсолютно непрерьвна на $[0, T]$ и для формы $a_{1}(t, u, v)=\partial a(t, u, v) / \partial t$ справедлива оценка

$$
\left|a_{1}(t, u, v)\right| \leqslant M_{2}\|u\|_{V}\|v\|_{V} .
$$

Здесь и далее производные понимаются в обобщенном смысле.

Форма $a(t, u, v)$ порождает линейньй ограниченньй из $V$ в $V^{\prime}$ оператор $A(t)$, определяемьй соотношением $a(t, u, v)=(A(t) u, v)$. Из (1.1) следует оценка $\|A(t) u\|_{V^{\prime}} \leqslant$ $M_{1}\|u\|_{V}$. Для всех $u \in V$ функция $t \rightarrow A(t) u \in V^{\prime}$ дифференцируема и $a_{1}(t, u, v)=$ $\left(A^{\prime}(t) u, v\right)$. Функция $t \rightarrow A^{\prime}(t) u \in V^{\prime}$ измерима. Из (1.2) почти при всех $t \in[0, T]$ следует оценка $\left\|A^{\prime}(t) u\right\|_{V^{\prime}} \leqslant M_{2}\|u\|_{V}$.

Для $t \in[0, T]$ определим множество

$$
D[A(t)]=\{u \in V \mid A(t) u \in H\} .
$$

Предположим существование гильбертова пространства $E$ такого, что для $t \in[0, T]$ выполняется $D[A(t)] \subset E \subset V$, пространство $V$ совпадает с интерполяционным пространством $[E, H]_{1 / 2}$ (см. $[8$, с. 23]) и для $t \in[0, T]$ и $u \in D[A(t)]$ вьполняется оценка $\|u\|_{E} \leqslant d\|A(t) u\|$.

Примеры реализации пространств $H, V$ и $E$ можно найти, например, в [1], [4], [8].

Предположим, что на $V$ для $t \in[0, T]$ определены линейные операторы $B(t): V \rightarrow H$ такие, что

$$
\|B(t) u\| \leqslant M_{3}\|u\|_{V}, \quad u \in V,
$$

для $u \in V$ функция $t \rightarrow B(t) u \in H$ дифференцируема и справедлива оценка $\left\|B^{\prime}(t) u\right\| \leqslant$ $M_{4}\|u\|_{V}$.

Предположим, что задана непрерьвная на $[0, T]$ функция $t \rightarrow f(t) \in H$ такая, что существует производная $f^{\prime} \in L_{2}\left(0, T ; V^{\prime}\right)$. Пусть также задан элемент $u^{0} \in D[A(0)]$.

В сделанных предположениях рассмотрим в пространстве $V^{\prime}$ задачу

$$
u^{\prime}(t)+[A(t)+B(t)] u(t)=f(t), \quad u(0)=u^{0} .
$$

В [9] установлено, что задача (1.4) имеет единственное решение $u(t)$, далее называемое гладким, такое, что

$$
u^{\prime} \in C([0, T], H) \cap L_{2}(0, T ; V), \quad A(\cdot) u(\cdot) \in C([0, T], H), \quad u^{\prime \prime} \in L_{2}\left(0, T ; V^{\prime}\right),
$$

и удовлетворяются уравнение и начальное условие (1.4). Из уравнения (1.4) следует, что и $B(\cdot) u(\cdot) \in C([0, T], H)$.

Отметим, что к (1.4) сводятся параболические задачи как с краевыми условиями Дирихле, так и Неймана, а также и смешанными краевыми условиями. Кроме того, можно рассматривать задачи произвольного $2 m, m \geqslant 1$, порядка по пространственным переменным (см., например, [8]). 
2. Проекционно-разностный метод с неявной схемой Эйлера по времени. Через $V_{h}$, где параметр $h>0$, обозначим конечномерное подпространство пространства $V$. Определим на элементах $u_{h} \in V_{h}$ двойственную норму $\left\|u_{h}\right\|_{V_{h}^{\prime}}=\sup \mid\left(u_{h}\right.$, $\left.v_{h}\right) \mid$, где точная верхняя грань берется по всем $v_{h} \in V_{h}$ c $\left\|v_{h}\right\|_{V}=1$. Очевидно, что $\left\|u_{h}\right\|_{V_{h}^{\prime}} \leqslant\left\|u_{h}\right\|_{V^{\prime}}$. Пусть $P_{h}$ - ортопроектор в пространстве $H$ на $V_{h}$. В [10] показано, что оператор $P_{h}$ допускает расширение по непрерьвности до оператора $\bar{P}_{h}: V^{\prime} \rightarrow V_{h}^{\prime}$ и $\left\|\bar{P}_{h} u\right\|_{V_{h}^{\prime}} \leqslant\|u\|_{V^{\prime}}$ для $u \in V^{\prime}$. Отметим для $u \in V^{\prime}$ и $v \in H$ соотношение $\left(\bar{P}_{h} u, v\right)=$ $\left(u, P_{h} v\right)[3]$.

Задаче (1.4) сопоставим разностную задачу в $V_{h}$ :

$$
\left(u_{k}^{h}-u_{k-1}^{h}\right) \tau^{-1}+\bar{P}_{h} A\left(t_{k}\right) u_{k}^{h}+P_{h} B\left(t_{k}\right) u_{k}^{h}=P_{h} f\left(t_{k}\right), \quad k=\overline{1, N},
$$

где $N$ - натуральное число, $\tau N=T, t_{k}=k \tau$ и элемент $u_{0}^{h} \in V_{h}$ считаем заданным. Задача (2.1) для достаточно малых $\tau$ однозначно разрешима [1].

В предположении, что подпространства $V_{h}$ такие, что

$$
\left\|\left(I-Q_{h}\right) v\right\|_{V} \leqslant c h\|v\|_{E}, \quad v \in E,
$$

где $Q_{h}$ - ортопроектор пространства $V$ на $V_{h}$, в [1] установлена оценка погрешности

$$
\begin{aligned}
& \max _{1 \leqslant k \leqslant N}\left\|u\left(t_{k}\right)-u_{k}^{h}\right\|_{V}^{2}+\sum_{k=1}^{N}\left\|\frac{1}{\tau} \int_{t_{k-1}}^{t_{k}} u^{\prime}(t) d t-\frac{u_{k}^{h}-u_{k-1}^{h}}{\tau}\right\|^{2} \tau \\
&+\sum_{k=1}^{N}\left\|\int_{t_{k-1}}^{t_{k}}\left(u^{\prime}(t)-\frac{u_{k}^{h}-u_{k-1}^{h}}{\tau}\right) d t\right\|_{V}^{2} \\
& \leqslant M\left\{\left\|Q_{h} u^{0}-u_{0}^{h}\right\|_{V}^{2}+\tau \int_{0}^{T}\left(\|u(t)\|_{V}^{2}+\left\|u^{\prime}(t)\right\|_{V}^{2}+\left\|u^{\prime \prime}(t)\right\|_{V^{\prime}}^{2}\right) d t\right. \\
&\left.+h^{2}\left(\max _{0 \leqslant t \leqslant T}\|A(t) u(t)\|^{2}+\int_{0}^{T}\left\|u^{\prime}(t)\right\|_{V}^{2} d t\right)\right\} .
\end{aligned}
$$

Простой анализ доказательства в [1] оценки (2.3) показывает, что при условии дополнительной гладкости решения задачи (1.4), $u^{\prime \prime} \in L_{p}(0, T ; H)$ для $1 \leqslant p \leqslant 2$, оценка погрешности следующая:

$$
\begin{aligned}
& \max _{1 \leqslant k \leqslant N}\left\|u\left(t_{k}\right)-u_{k}^{h}\right\|_{V}^{2}+\sum_{k=1}^{N}\left\|\frac{1}{\tau} \int_{t_{k-1}}^{t_{k}} u^{\prime}(t) d t-\frac{u_{k}^{h}-u_{k-1}^{h}}{\tau}\right\|^{2} \tau \\
&+\sum_{k=1}^{N}\left\|u^{\prime}\left(t_{k}\right)-\frac{u_{k}^{h}-u_{k-1}^{h}}{\tau}\right\|^{2} \tau \\
& \leqslant M\left\{\left\|Q_{h} u^{0}-u_{0}^{h}\right\|_{V}^{2}+\tau^{3-2 / p}\left(\int_{0}^{T}\left\|u^{\prime \prime}(t)\right\|^{p} d t\right)^{2 / p}\right. \\
&\left.+h^{2}\left(\max _{0 \leqslant t \leqslant T}\|A(t) u(t)\|^{2}+\int_{0}^{T}\left\|u^{\prime}(t)\right\|_{V}^{2} d t\right)\right\} .
\end{aligned}
$$


При получении оценки (2.4) главное в работе [1] в тождестве (14) иначе оценить слагаемое

$$
\begin{aligned}
I_{6} & =\frac{2}{\tau^{2}} \int_{t_{k-1}}^{t_{k}}\left(\int_{t_{k}}^{t} \operatorname{Re}\left(u^{\prime \prime}(s), z_{k}^{h}-z_{k-1}^{h}\right) d s\right) d t \\
& \leqslant \varepsilon^{-1}\left(\int_{t_{k-1}}^{t_{k}}\left\|u^{\prime \prime}(s)\right\| d s\right)^{2}+\varepsilon\left\|\left(z_{k}^{h}-z_{k-1}^{h}\right) \tau^{-1}\right\|^{2} \\
& \leqslant \varepsilon^{-1} \tau^{2-2 / p}\left(\int_{t_{k-1}}^{t_{k}}\left\|u^{\prime \prime}(t)\right\|^{p} d t\right)^{2 / p}+\varepsilon\left\|\left(z_{k}^{h}-z_{k-1}^{h}\right) \tau^{-1}\right\|^{2}
\end{aligned}
$$

где $\varepsilon>0$ и достаточно мало.

Далее покажем, что более слабая норма погрешности, а именно, выражение

$$
S_{1} \equiv \max _{1 \leqslant k \leqslant N}\left\|u\left(t_{k}\right)-u_{k}^{h}\right\|^{2}+\sum_{k=1}^{N}\left\|\int_{t_{k-1}}^{t_{k}}\left(u^{\prime}(t)-\frac{u_{k}^{h}-u_{k-1}^{h}}{\tau}\right) d t\right\|^{2}
$$

при $h \rightarrow 0$ и $\tau \rightarrow 0$ стремится к нулю по параметру $h$, вообще говоря, быстрее, чем это следует из оценок (2.3) и (2.4).

Предположим дополнительно, что в задаче (1.4) операторы $B(t): H \rightarrow V^{\prime}$ и справедлива оценка

$$
\|B(t) u\|_{V^{\prime}} \leqslant M_{4}\|u\|, \quad u \in H .
$$

В приложениях условия (1.3) и (2.6) фактически выполняются одновременно.

Как в [1] и [2], определим для $t \in[0, T]$ гильбертовы пространства $V(t)$, задав на $V$ новое скалярное произведение,

$$
V(t)=\left\{u, v \in V \mid(u, v)_{V(t)}=a(t, u, v)\right\}
$$

Через $Q_{h}(t)$ обозначим ортопроектор в пространстве $V(t)$ на $V_{h}$.

ТЕОРемА 1. Пусть для задачи (1.4) выполнены перечисленные выше условия гладкой разрешимости. Пусть, кроме того, операторы $B(t)$ удовлетворяют условию (2.6) и $и(t)$ - решение задачи (1.4) такое, что $u^{\prime \prime}(t)=u_{1}^{\prime \prime}(t)+u_{2}^{\prime \prime}(t)$, әде $u_{1}^{\prime \prime} \in L_{p}(0, T ; H)$ для $1 \leqslant p \leqslant 2 u u_{2}^{\prime \prime} \in L_{2}\left(0, T ; V^{\prime}\right)$. Пусть, наконеи, $u_{k}^{h}$ - решение задачи (2.1). Тогда справедлива оченка

$$
\begin{aligned}
\max _{1 \leqslant k \leqslant N} & \left\|u\left(t_{k}\right)-u_{k}^{h}\right\|^{2}+\sum_{k=1}^{N}\left\|\int_{t_{k-1}}^{t_{k}}\left(u^{\prime}(t)-\frac{u_{k}^{h}-u_{k-1}^{h}}{\tau}\right) d t\right\|^{2} \\
& +\sum_{k=1}^{N}\left\|Q_{h}\left(t_{k}\right) u\left(t_{k}\right)-u_{k}^{h}\right\|_{V}^{2} \tau \\
\leqslant & M\left\{\left\|u^{0}-u_{0}^{h}\right\|^{2}+\tau^{3-2 / p}\left(\int_{0}^{T}\left\|u_{1}^{\prime \prime}(t)\right\|^{p} d t\right)^{2 / p}+\tau^{2} \int_{0}^{T}\left\|u_{2}^{\prime \prime}(t)\right\|_{V^{\prime}}^{2} d t\right. \\
& \left.+\left(\sum_{k=1}^{N}\left\|\frac{1}{\tau} \int_{t_{k-1}}^{t_{k}} u^{\prime}(t) d t-\frac{u_{k}^{h}-u_{k-1}^{h}}{\tau}\right\|^{2} \tau\right)^{1 / 2}\left(\sum_{k=1}^{N}\left\|\left[I-Q_{h}\left(t_{k}\right)\right] u\left(t_{k}\right)\right\|^{2} \tau\right)^{1 / 2}\right\} .
\end{aligned}
$$


ДокАЗАТЕЛЬСТво. Обозначим $z_{k}=u\left(t_{k}\right)-u_{k}^{h}$. Тогда, учитывая свойство

$$
\bar{P}_{h} A(t) u=\bar{P}_{h} A(t) Q_{h}(t) u, \quad \text { где } u \in V,
$$

нетрудно установить тождество

$$
\left(z_{k}-z_{k-1}\right) \tau^{-1}+\bar{P}_{h} A\left(t_{k}\right) z_{k}^{h}+P_{h} B\left(t_{k}\right) z_{k}=\left\{\left[u\left(t_{k}\right)-u\left(t_{k-1}\right)\right] \tau^{-1}-P_{h} u^{\prime}\left(t_{k}\right)\right\},
$$

где $z_{k}^{h}=Q_{h}\left(t_{k}\right) u\left(t_{k}\right)-u_{k}^{h}$. Умножим (2.8) скалярно в $H$ на $z_{k}^{h}$. После простых преобразований получим

$$
\begin{aligned}
& \left(\left(z_{k}-z_{k-1}\right) \tau^{-1}, z_{k}\right)+a\left(t_{k}, z_{k}^{h}, z_{k}^{h}\right)=\left(\left(z_{k}-z_{k-1}\right) \tau^{-1},\left[I-Q_{h}\left(t_{k}\right)\right] u\left(t_{k}\right)\right) \\
& -\left(B\left(t_{k}\right) z_{k}, z_{k}^{h}\right)+\left(\frac{1}{\tau} \int_{t_{k-1}}^{t_{k}}\left[u^{\prime}(t)-u^{\prime}\left(t_{k}\right)\right] d t, z_{k}^{h}\right) .
\end{aligned}
$$

В равенстве (2.9) возьмем удвоенную вещественную часть и воспользуемся оценкой (1.1). Получим неравенство

$$
\begin{aligned}
& \left\|z_{k}\right\|^{2} \tau^{-1}-\left\|z_{k-1}\right\|^{2} \tau^{-1}+\left\|z_{k}-z_{k-1}\right\|^{2} \tau^{-1}+2 \delta\left\|z_{k}^{h}\right\|_{V}^{2} \\
& \leqslant 2 \operatorname{Re}\left(\left(z_{k}-z_{k-1}\right) \tau^{-1},\left[I-Q_{h}\left(t_{k}\right)\right] u\left(t_{k}\right)\right)-2 \operatorname{Re}\left(B\left(t_{k}\right) z_{k}, z_{k}^{h}\right) \\
& \quad+2 \operatorname{Re}\left(\frac{1}{\tau} \int_{t_{k-1}}^{t_{k}}\left[u^{\prime}(t)-u^{\prime}\left(t_{k}\right)\right] d t, z_{k}^{h}\right)=\sum_{i=1}^{3} I_{i} .
\end{aligned}
$$

Оцениваем слагаемые $I_{i}, i=1,2$, в правой части (2.10). Имеем

$$
\begin{aligned}
& I_{1} \leqslant 2\left\|\left(z_{k}-z_{k-1}\right) \tau^{-1}\right\|\left\|\left[I-Q_{h}\left(t_{k}\right)\right] u\left(t_{k}\right)\right\|, \\
& I_{2} \leqslant 2 M_{4}\left\|z_{k}\right\|\left\|z_{k}^{h}\right\|_{V} \leqslant \varepsilon_{1}\left\|z_{k}^{h}\right\|_{V}^{2}+\varepsilon_{1}^{-1} M_{4}^{2}\left\|z_{k}\right\|^{2} .
\end{aligned}
$$

Слагаемое $I_{3}$ в $(2.10)$ преобразуем так:

$$
I_{3}=\frac{2}{\tau} \operatorname{Re} \int_{t_{k-1}}^{t_{k}}\left(\int_{t_{k}}^{t} u_{1}^{\prime \prime}(s) d s, z_{k}^{h}\right) d t+\frac{2}{\tau} \operatorname{Re} \int_{t_{k-1}}^{t_{k}}\left(\int_{t_{k}}^{t} u_{2}^{\prime \prime}(s) d s, z_{k}^{h}\right) d t=I_{3}^{1}+I_{3}^{2} .
$$

Оцениваем $I_{3}^{1}$ :

$$
\begin{aligned}
I_{3}^{1} & \leqslant 2 \int_{t_{k-1}}^{t_{k}}\left\|u_{1}^{\prime \prime}(s)\right\| d s\left\|z_{k}^{h}\right\| \leqslant 2 c \int_{t_{k-1}}^{t_{k}}\left\|u_{1}^{\prime \prime}(s)\right\| d s\left\|z_{k}^{h}\right\|_{V} \\
& \leqslant \varepsilon_{2}^{-1} c^{2} \tau^{2-2 / p}\left(\int_{t_{k-1}}^{t_{k}}\left\|u_{1}^{\prime \prime}(s)\right\|^{p} d s\right)^{2 / p}+\varepsilon_{2}\left\|z_{k}^{h}\right\|_{V}^{2} .
\end{aligned}
$$

Для слагаемого $I_{3}^{2}$ получим

$$
I_{3}^{2} \leqslant 2 \int_{t_{k-1}}^{t_{k}}\left\|u_{2}^{\prime \prime}(s)\right\|_{V^{\prime}} d s\left\|z_{k}^{h}\right\|_{V} \leqslant \varepsilon_{3}^{-1} \tau \int_{t_{k-1}}^{t_{k}}\left\|u_{2}^{\prime \prime}(s)\right\|_{V^{\prime}}^{2} d s+\varepsilon_{3}\left\|z_{k}^{h}\right\|_{V}^{2} .
$$


Положим $\varepsilon_{1}=\varepsilon_{2}=\varepsilon_{3}=\delta / 3$. В результате из (2.10) следует оценка

$$
\begin{aligned}
& \left\|z_{k}\right\|^{2} \tau^{-1}-\left\|z_{k-1}\right\|^{2} \tau^{-1}+\left\|z_{k}-z_{k-1}\right\|^{2} \tau^{-1}+\delta\left\|z_{k}^{h}\right\|_{V}^{2} \\
& \leqslant 2\left\|\left(z_{k}-z_{k-1}\right) \tau^{-1}\right\|\left\|\left[I-Q_{h}\left(t_{k}\right)\right] u\left(t_{k}\right)\right\| \\
& \quad+c_{1} \tau^{2-2 / p}\left(\int_{t_{k-1}}^{t_{k}}\left\|u_{1}^{\prime \prime}(t)\right\|^{p} d t\right)^{2 / p}+c_{2} \tau \int_{t_{k-1}}^{t_{k}}\left\|u_{2}^{\prime \prime}(t)\right\|_{V^{\prime}}^{2} d t+c_{3}\left\|z_{k}\right\|^{2} .
\end{aligned}
$$

Умножим (2.11) на $\tau$ и просуммируем по $k$ от 1 до $m \leqslant N$. Имеем

$$
\begin{aligned}
& \left\|z_{m}\right\|^{2}+\sum_{k=1}^{m}\left(\left\|z_{k}-z_{k-1}\right\|^{2}+\delta\left\|z_{k}^{h}\right\|_{V}^{2} \tau\right) \\
& \leqslant\left\|z_{0}\right\|^{2}+2\left(\sum_{k=1}^{N}\left\|\left(z_{k}-z_{k-1}\right) \tau^{-1}\right\|^{2} \tau\right)^{1 / 2}\left(\sum_{k=1}^{N}\left\|\left[I-Q_{h}\left(t_{k}\right)\right] u\left(t_{k}\right)\right\|^{2} \tau\right)^{1 / 2} \\
& \quad+c_{1} \tau^{3-2 / p}\left(\int_{0}^{T}\left\|u_{1}^{\prime \prime}(t)\right\|^{p} d t\right)^{2 / p}+c_{2} \tau^{2} \int_{0}^{T}\left\|u_{2}^{\prime \prime}(t)\right\|_{V^{\prime}}^{2} d t+c_{3} \tau \sum_{k=1}^{m}\left\|z_{k}\right\|^{2} .
\end{aligned}
$$

В (2.12) выделяем суммарное неравенство для $\left\|z_{m}\right\|^{2}$. Получим оценку (2.7) для $\max \left\|u\left(t_{k}\right)-u_{k}^{h}\right\|^{2}$. Эту оценку подставим в (2.12) и получим (2.7) в полном объеме.

ЗАмечание 1 . Если в задаче $(1.4) f^{\prime} \in L_{2}\left(0, T ; V^{\prime}\right)$, то решение $u(t)$ таково, что $u^{\prime \prime} \in L_{2}\left(0, T ; V^{\prime}\right)$, и оценка $(2.7)$ вьполняется с $u_{1}^{\prime \prime}(t)=0$ и $u_{2}^{\prime \prime}(t)=u^{\prime \prime}(t)$. Если же решение $u(t)$ задачи (1.4) такое, что $u^{\prime \prime} \in L_{p}(0, T ; H)$ для $1 \leqslant p \leqslant 2$, то оценка (2.7) выполняется с $u_{1}^{\prime \prime}(t)=u^{\prime \prime}(t)$ и $u_{2}^{\prime \prime}(t)=0$.

Обратим теперь внимание, что в (2.3) и (2.4) установлены оценки выражения

$$
\sum_{k=1}^{N}\left\|\frac{1}{\tau} \int_{t_{k-1}}^{t_{k}} u^{\prime}(t) d t-\frac{u_{k}^{h}-u_{k-1}^{h}}{\tau}\right\|^{2} \tau,
$$

которые можно подставить в оценку (2.7). В результате получим

СлЕДСТВИЕ 1. Пусть выполнены условия гладкой разрешимости задачи (1.4) $и$ условие (2.2), т.е. выполняется оценка (2.7). Пусть, кроме того, операторы $B(t)$ удовлетворяют условию (2.6). Тогда справедлива оценка

$$
\begin{aligned}
S_{1} \leqslant M & \left\{\left\|Q_{h} u^{0}-u_{0}^{h}\right\|_{V}^{2}+\tau \int_{0}^{T}\left(\|u(t)\|_{V}^{2}+\left\|u^{\prime}(t)\right\|_{V}^{2}+\left\|u^{\prime \prime}(t)\right\|_{V^{\prime}}^{2}\right) d t\right. \\
& \left.+h^{3}\left(\max _{0 \leqslant t \leqslant T}\|A(t) u(t)\|^{2}+\int_{0}^{T}\left\|u^{\prime}(t)\right\|_{V}^{2} d t\right)\right\} .
\end{aligned}
$$

Если же дополнительно решение $u(t)$ такое, что $u^{\prime \prime} \in L_{p}(0, T ; H)$ для $1 \leqslant p \leqslant 2$, т.е. выполняется оценка (2.4), то

$$
\begin{aligned}
S_{1} \leqslant M & \left\{\left\|Q_{h} u^{0}-u_{0}^{h}\right\|_{V}^{2}+\tau^{3-2 / p}\left(\int_{0}^{T}\left\|u^{\prime \prime}(t)\right\|^{p} d t\right)^{2 / p}\right. \\
& \left.+h^{3}\left(\max _{0 \leqslant t \leqslant T}\|A(t) u(t)\|^{2}+\int_{0}^{T}\left\|u^{\prime}(t)\right\|_{V}^{2} d t\right)\right\} .
\end{aligned}
$$

$B(2.14) u(2.15)$ вырахсение $S_{1}$ определено в (2.5). 
ДокАЗАТЕЛЬСТво. Прежде всего, заметим, что

$$
\begin{aligned}
\left\|u^{0}-u_{h}^{0}\right\|_{H}^{2} & \leqslant 2\left\|u^{0}-Q_{h} u^{0}\right\|_{H}^{2}+2\left\|Q_{h} u^{0}-u_{h}^{0}\right\|_{H}^{2} \\
& \left.\leqslant c_{1} h^{4} \| A(0) u^{0}\right)\left\|_{H}^{2}+c_{2}\right\| Q_{h} u^{0}-u_{h}^{0} \|_{V}^{2} .
\end{aligned}
$$

Следует также воспользоваться оценкой

$$
\left(\sum_{k=1}^{N}\left\|\left[I-Q_{h}\left(t_{k}\right)\right] u\left(t_{k}\right)\right\|^{2} \tau\right)^{1 / 2} \leqslant c h^{2} \max _{0 \leqslant t \leqslant T}\|A(t) u(t)\|,
$$

обоснованной, например, в [4]. Затем оценки (2.4) и (2.5) выражения (2.13) нужно подставить в (2.7). После несложных преобразований получим (2.14) и (2.15).

3. Проекционно-разностный метод с модифицированной схемой Кранка-Николсон по времени. В качестве задачи, приближенной к (1.4), которая удовлетворяет условиям гладкой разрешимости, рассмотрим разностную задачу в $V_{h}$

$$
\begin{aligned}
& \left(u_{k}^{h}-u_{k-1}^{h}\right) \tau_{k}^{-1}+\bar{P}_{h}\left[A\left(t_{k}\right) u_{k}^{h}+A\left(t_{k-1}\right) u_{k-1}^{h}\right] 2^{-1} \\
& \quad+P_{h}\left[B\left(t_{k}\right) u_{k}^{h}+B\left(t_{k-1}\right) u_{k-1}^{h}\right] 2^{-1}=f_{h}^{k}, \quad k=\overline{1, N}
\end{aligned}
$$

где элемент $u_{0}^{h} \in V_{h}$ считаем заданным, $f_{h}^{k}=P_{h}\left[f\left(t_{k}\right)+f\left(t_{k-1}\right)\right] 2^{-1}$, а остальные параметры задачи (3.1) такие же, как в задаче (2.1). Как и задача (2.1), задача (3.1) для достаточно малых $\tau$ однозначно разрешима [2].

В [2] установлены оценки для погрешности

$$
S_{2} \equiv \max _{1 \leqslant k \leqslant N}\left\|u\left(t_{k}\right)-u_{k}^{h}\right\|_{V}^{2}+\sum_{k=1}^{N}\left\|\frac{1}{\tau} \int_{t_{k-1}}^{t_{k}} u^{\prime}(t) d t-\frac{u_{k}^{h}-u_{k-1}^{h}}{\tau}\right\|^{2} \tau .
$$

В предположении (2.2) и в предположении дополнительной гладкости решения задачи (1.4) $u^{\prime \prime} \in L_{p}(0, T ; H)$ для некоторого $1 \leqslant p \leqslant 2$, справедлива оценка

$$
\begin{aligned}
S_{2} \leqslant & M\left\{\left\|Q_{h} u^{0}-u_{0}^{h}\right\|_{V}^{2}+h^{2}\left[\max _{0 \leqslant t \leqslant T}\|A(t) u(t)\|^{2}+\int_{0}^{T}\left\|u^{\prime}(t)\right\|_{V}^{2} d t\right]\right. \\
& \left.+\tau^{3-2 / p}\left(\int_{0}^{T}\left\|u^{\prime \prime}(t)\right\|^{p} d t\right)^{2 / p}\right\},
\end{aligned}
$$

где погрешность $S_{2}$ определена в (3.2). Если же существует $u^{\prime \prime \prime} \in L_{p}(0, T ; H)$ для некоторого $1 \leqslant p \leqslant 2$, то для $S_{2}$ справедлива оценка

$$
\begin{aligned}
S_{2} \leqslant & M\left\{\left\|Q_{h} u^{0}-u_{0}^{h}\right\|_{V}^{2}+h^{2}\left[\max _{0 \leqslant t \leqslant T}\|A(t) u(t)\|^{2}+\int_{0}^{T}\left\|u^{\prime}(t)\right\|_{V}^{2} d t\right]\right. \\
& \left.+\tau^{5-2 / p}\left(\int_{0}^{T}\left\|u^{\prime \prime \prime}(t)\right\|^{p} d t\right)^{2 / p}\right\} .
\end{aligned}
$$

Далее покажем, что выражение $\max _{1 \leqslant k \leqslant N}\left\|u\left(t_{k}\right)-u_{k}^{h}\right\|^{2}$ при $h \rightarrow 0$ и $\tau \rightarrow 0$ стремится к нулю по параметру $h$, вообще говоря, быстрее, чем это следует из оценок $(3.3)$ и (3.4). 
ТЕОРема 2. Пусть для задачи (1.4) выполнены перечисленные выше условия гладкой разрешимости. Кроме того, операторы $B(t)$ удовлетворяют условию (2.6). Пусть $и(t)$ - решение задачи (1.4), а $u_{k}^{h}$ - решение задачи (3.1). Тогда справедлива оченка

$$
\begin{aligned}
\max _{1 \leqslant k \leqslant N} & \left\|u\left(t_{k}\right)-u_{k}^{h}\right\|^{2} \\
\leqslant M & \left\{\left\|u^{0}-u_{0}^{h}\right\|^{2}+\frac{1}{\tau} \sum_{k=1}^{N}\left\|\int_{t_{k-1}}^{t_{k}}\left(\frac{u^{\prime}\left(t_{k}\right)+u^{\prime}\left(t_{k-1}\right)}{2}-u^{\prime}(t)\right) d t\right\|^{2}\right. \\
& +\tau^{2}\left[\left\|Q_{h} u^{0}-u_{0}^{h}\right\|_{V}^{2}+\max _{0 \leqslant t \leqslant T}\left\|\left(I-Q_{h}\right) u(t)\right\|_{V}^{2}+\int_{0}^{T}\left\|\left[I-Q_{h}(t)\right] u^{\prime}(t)\right\|^{2} d t\right] \\
& \left.+\left(\sum_{k=1}^{N}\left\|\frac{1}{\tau} \int_{t_{k-1}}^{t_{k}} u^{\prime}(t) d t-\frac{u_{k}^{h}-u_{k-1}^{h}}{\tau}\right\|^{2} \tau\right)^{1 / 2} \max _{0 \leqslant t \leqslant T}\left\|\left[I-Q_{h}(t)\right] u(t)\right\|\right\} .
\end{aligned}
$$

ДокАЗАТЕЛЬСтво. Обозначим $z_{k}=u\left(t_{k}\right)-u_{k}^{h}$. Тогда нетрудно установить равенства

$$
\begin{aligned}
\left(z_{k}\right. & \left.-z_{k-1}\right) \tau^{-1}+\bar{P}_{h}\left[A\left(t_{k}\right) z_{k}^{h}+A\left(t_{k-1}\right) z_{k-1}^{h}\right] 2^{-1}+P_{h}\left[B\left(t_{k}\right) z_{k}+B\left(t_{k-1}\right) z_{k-1}\right] 2^{-1} \\
& =\left[u\left(t_{k}\right)-u\left(t_{k-1}\right)\right] \tau^{-1}-P_{h}\left[u^{\prime}\left(t_{k}\right)+u^{\prime}\left(t_{k-1}\right)\right] 2^{-1}
\end{aligned}
$$

где $z_{k}^{h}=Q_{h}\left(t_{k}\right) u\left(t_{k}\right)-u_{k}^{h}$ и $k=\overline{1, N}$. Умножим $(3.6)$ на $\left(z_{k}^{h}+z_{k-1}^{h}\right) \tau$ скалярно в $H$. В результате после некоторых преобразований получим

$$
\begin{aligned}
& \left(z_{k}-z_{k-1}, z_{k}+z_{k-1}\right)+2\left(\frac{A\left(t_{k}\right) z_{k}^{h}+A\left(t_{k-1}\right) z_{k-1}^{h}}{2}, \frac{z_{k}^{h}+z_{k-1}^{h}}{2}\right) \tau \\
& =\left(\left(z_{k}-z_{k-1}\right) \tau^{-1},\left[I-Q_{h}\left(t_{k}\right)\right] u\left(t_{k}\right)+\left[I-Q_{h}\left(t_{k-1}\right)\right] u\left(t_{k-1}\right)\right) \tau \\
& \quad+2\left(\frac{u\left(t_{k}\right)-u\left(t_{k-1}\right)}{\tau}-\frac{u^{\prime}\left(t_{k}\right)+u^{\prime}\left(t_{k-1}\right)}{2}, \frac{z_{k}^{h}+z_{k-1}^{h}}{2}\right) \tau \\
& \quad-2\left(\frac{B\left(t_{k}\right) z_{k}+B\left(t_{k-1}\right) z_{k-1}}{2}, \frac{z_{k}^{h}+z_{k-1}^{h}}{2}\right) \tau .
\end{aligned}
$$

Преобразуем здесь выражение

$$
\frac{A\left(t_{k}\right) z_{k}^{h}+A\left(t_{k-1}\right) z_{k-1}^{h}}{2}=A\left(t_{k}\right) \frac{z_{k}^{h}+z_{k-1}^{h}}{2}+\frac{\left[A\left(t_{k-1}\right)-A\left(t_{k}\right)\right] z_{k-1}^{h}}{2} .
$$

Учитьвая последнее соотношение, возьмем от (3.7) вещественную часть:

$$
\begin{aligned}
\left\|z_{k}\right\|^{2} & -\left\|z_{k-1}\right\|^{2}+2\left(A\left(t_{k}\right) \frac{z_{k}^{h}+z_{k-1}^{h}}{2}, \frac{z_{k}^{h}+z_{k-1}^{h}}{2}\right) \tau \\
= & 2 \operatorname{Re}\left(\left(z_{k}-z_{k-1}\right) \tau^{-1},\left[I-Q_{h}\left(t_{k}\right)\right] u\left(t_{k}\right)+\left[I-Q_{h}\left(t_{k-1}\right)\right] u\left(t_{k-1}\right)\right) \tau \\
& +2 \operatorname{Re}\left(\frac{u\left(t_{k}\right)-u\left(t_{k-1}\right)}{\tau}-\frac{u^{\prime}\left(t_{k}\right)+u^{\prime}\left(t_{k-1}\right)}{2}, \frac{z_{k}^{h}+z_{k-1}^{h}}{2}\right) \tau \\
& -2 \operatorname{Re}\left(\frac{B\left(t_{k}\right) z_{k}+B\left(t_{k-1}\right) z_{k-1}}{2}, \frac{z_{k}^{h}+z_{k-1}^{h}}{2}\right) \tau \\
& +\operatorname{Re}\left(\left[A\left(t_{k}\right)-A\left(t_{k-1}\right)\right] z_{k-1}^{h},\left(z_{k}^{h}+z_{k-1}^{h}\right) 2^{-1}\right) \tau=\sum_{i=1}^{4} I_{i} .
\end{aligned}
$$


Оценим слагаемые в обеих частях (3.8). Прежде всего, из (1.1) получим

$$
2\left(A\left(t_{k}\right) \frac{z_{k}^{h}+z_{k-1}^{h}}{2}, \frac{z_{k}^{h}+z_{k-1}^{h}}{2}\right) \tau \geqslant 2 \delta\left\|\frac{z_{k}^{h}+z_{k-1}^{h}}{2}\right\|_{V}^{2} .
$$

Теперь оцениваем сверху слагаемые $I_{i}$ в правой части (3.8):

$$
I_{1} \leqslant 2\left\|\left(z_{k}-z_{k-1}\right) \tau^{-1}\right\| 2 \max _{0 \leqslant t \leqslant T}\left\|\left[I-Q_{h}(t)\right] u(t)\right\| \tau .
$$

Слагаемое $I_{2}$ прежде преобразуем:

$$
\begin{aligned}
I_{2} & =2 \operatorname{Re}\left(\int_{t_{k-1}}^{t_{k}}\left(u^{\prime}(t)-\frac{u^{\prime}\left(t_{k}\right)+u^{\prime}\left(t_{k-1}\right)}{2}\right) d t, \frac{z_{k}^{h}+z_{k-1}^{h}}{2}\right) \\
& \leqslant 2\left\|\int_{t_{k-1}}^{t_{k}}\left(u^{\prime}(t)-\frac{u^{\prime}\left(t_{k}\right)+u^{\prime}\left(t_{k-1}\right)}{2}\right) d t\right\|_{V^{\prime}}\left\|\frac{z_{k}^{h}+z_{k-1}^{h}}{2}\right\|_{V} \\
& \leqslant \frac{1}{\varepsilon_{1} \tau}\left\|\int_{t_{k-1}}^{t_{k}}\left(u^{\prime}(t)-\frac{u^{\prime}\left(t_{k}\right)+u^{\prime}\left(t_{k-1}\right)}{2}\right) d t\right\|_{V^{\prime}}^{2}+\varepsilon_{1}\left\|\frac{z_{k}^{h}+z_{k-1}^{h}}{2}\right\|_{V}^{2} \tau .
\end{aligned}
$$

При оценке слагаемого $I_{3}$ воспользуемся условием (2.6):

$$
\begin{aligned}
I_{3} & \leqslant \varepsilon_{2}^{-1}\left\|\frac{B\left(t_{k}\right) z_{k}+B\left(t_{k-1}\right) z_{k-1}}{2}\right\|_{V^{\prime}}^{2} \tau+\varepsilon_{2}\left\|\frac{z_{k}^{h}+z_{k-1}^{h}}{2}\right\|_{V}^{2} \tau \\
& \leqslant \varepsilon_{2}^{-1} 2^{-1} M_{4}^{2}\left(\left\|z_{k}\right\|^{2}+\left\|z_{k-1}\right\|^{2}\right) \tau+\varepsilon_{2}\left\|\frac{z_{k}^{h}+z_{k-1}^{h}}{2}\right\|_{V}^{2} \tau .
\end{aligned}
$$

Оценим, наконец,

$$
I_{4} \leqslant M_{2} \tau\left\|z_{k-1}^{h}\right\|_{V}\left\|\frac{z_{k}^{h}+z_{k-1}^{h}}{2}\right\|_{V} \tau \leqslant \varepsilon_{3}^{-1} M_{2}^{2} \tau^{3}\left\|z_{k-1}^{h}\right\|_{V}^{2}+\varepsilon_{3}\left\|\frac{z_{k}^{h}+z_{k-1}^{h}}{2}\right\|_{V}^{2} \tau .
$$

Положим $\varepsilon_{1}=\varepsilon_{2}=\varepsilon_{3}=\delta / 3$. Тогда из (3.8) получим оценку

$$
\begin{aligned}
\left\|z_{k}\right\|^{2} & -\left\|z_{k-1}\right\|^{2}+\delta\left\|\frac{z_{k}^{h}+z_{k-1}^{h}}{2}\right\|_{V}^{2} \tau \\
\leqslant & c_{1}\left\|z_{k}\right\|^{2} \tau+c_{2}\left\|z_{k-1}\right\|^{2} \tau+c_{3} \tau^{3}\left\|z_{k-1}^{h}\right\|_{V}^{2} \\
& +4\left\|\left(z_{k}-z_{k-1}\right) \tau^{-1}\right\| \max _{0 \leqslant t \leqslant T}\left\|\left[I-Q_{h}(t)\right] u(t)\right\| \tau \\
& +\frac{c_{4}}{\tau}\left\|\int_{t_{k-1}}^{t_{k}}\left(u^{\prime}(t)-\frac{u^{\prime}\left(t_{k}\right)+u^{\prime}\left(t_{k-1}\right)}{2}\right) d t\right\|_{V^{\prime}}^{2}
\end{aligned}
$$

Неравенства (3.9) суммируем по $k$ от 1 до $m \leqslant N$. В результате простых преобразований получим

$$
\begin{aligned}
\left\|z_{m}\right\|^{2} & +\delta \sum_{k=1}^{m}\left\|\frac{z_{k}^{h}+z_{k-1}^{h}}{2}\right\|_{V}^{2} \tau \\
\leqslant & c_{1}\left\{\left\|z_{0}\right\|^{2}+\tau^{3} \sum_{k=1}^{m}\left\|z_{k-1}^{h}\right\|_{V}^{2}\right. \\
& +\left(\sum_{k=1}^{m}\left\|\left(z_{k}-z_{k-1}\right) \tau^{-1}\right\|^{2} \tau\right)^{1 / 2} \max _{0 \leqslant t \leqslant T}\left\|\left[I-Q_{h}(t)\right] u(t)\right\| \\
& \left.+\frac{1}{\tau} \sum_{k=1}^{m}\left\|\int_{t_{k-1}}^{t_{k}}\left(u^{\prime}(t)-\frac{u^{\prime}\left(t_{k}\right)+u^{\prime}\left(t_{k-1}\right)}{2}\right) d t\right\|_{V^{\prime}}^{2}\right\}+c_{2} \tau \sum_{k=1}^{m}\left\|z_{k}^{h}\right\|^{2}
\end{aligned}
$$


В (3.10) рассмотрим слагаемое

$\tau^{3} \sum_{k=1}^{m}\left\|z_{k-1}^{h}\right\|_{V}^{2} \leqslant \tau^{2} T \max _{0 \leqslant k \leqslant N}\left\|z_{k}^{h}\right\|_{V}^{2} \leqslant \tau^{2} T\left(\left\|Q_{h}(0) u^{0}-u_{0}^{h}\right\|_{V}^{2}+\max _{1 \leqslant k \leqslant N}\left\|z_{k}^{h}\right\|_{V}^{2}\right)$.

Обратим внимание на соотношение $z_{k}^{h}=z_{k}-\left[I-Q_{h}\left(t_{k}\right)\right] u\left(t_{k}\right)$. Тогда получим

$$
\max _{1 \leqslant k \leqslant N}\left\|z_{k}^{h}\right\|_{V}^{2} \leqslant 2 \max _{1 \leqslant k \leqslant N}\left\|z_{k}\right\|_{V}^{2}+2 \max _{0 \leqslant t \leqslant T}\left\|\left[I-Q_{h}(t)\right] u(t)\right\|_{V}^{2}
$$

Воспользуемся теперь оценкой

$$
\left\|\left[I-Q_{h}(t)\right] v\right\|_{V}^{2} \leqslant M_{1} \delta^{-1}\left\|\left(I-Q_{h}\right) v\right\|_{V}^{2}, \quad v \in V
$$

а также оценкой, установленной в [2],

$$
\begin{aligned}
\max _{1 \leqslant k \leqslant N}\left\|z_{k}\right\|_{V}^{2} \leqslant & c\left\{\left\|Q_{h} u^{0}-u_{0}^{h}\right\|_{V}^{2}+\max _{0 \leqslant t \leqslant T}\left\|\left(I-Q_{h}\right) u(t)\right\|_{V}^{2}\right. \\
& +\int_{0}^{T}\left\|\left[I-Q_{h}(t)\right] u^{\prime}(t)\right\|^{2} d t \\
& \left.+\frac{1}{\tau} \sum_{k=1}^{N}\left\|\int_{t_{k-1}}^{t_{k}}\left(\frac{u^{\prime}\left(t_{k}\right)+u^{\prime}\left(t_{k-1}\right)}{2}-u^{\prime}(t)\right) d t\right\|^{2}\right\} .
\end{aligned}
$$

Тогда получим

$$
\begin{aligned}
\tau^{3} \sum_{k=1}^{m}\left\|z_{k-1}^{h}\right\|_{V}^{2} \leqslant & c \tau^{2}\left\{\left\|Q_{h}(0) u^{0}-u_{0}^{h}\right\|_{V}^{2}+\max _{0 \leqslant t \leqslant T}\left\|\left(I-Q_{h}\right) u(t)\right\|_{V}^{2}\right. \\
& +\int_{0}^{T}\left\|\left[I-Q_{h}(t)\right] u^{\prime}(t)\right\|^{2} d t \\
& \left.+\frac{1}{\tau} \sum_{k=1}^{N}\left\|\int_{t_{k-1}}^{t_{k}}\left(\frac{u^{\prime}\left(t_{k}\right)+u^{\prime}\left(t_{k-1}\right)}{2}-u^{\prime}(t)\right) d t\right\|^{2}\right\}
\end{aligned}
$$

Из (3.10) и (3.11) следует оценка

$$
\begin{aligned}
\left\|z_{m}\right\|^{2} \leqslant & c\left\{\left\|z_{0}\right\|^{2}+\left(\sum_{k=1}^{N}\left\|\left(z_{k}-z_{k-1}\right) \tau^{-1}\right\|^{2} \tau\right)^{1 / 2} \max _{0 \leqslant t \leqslant T}\left\|\left[I-Q_{h}(t)\right] u(t)\right\|\right. \\
& +\frac{1}{\tau} \sum_{k=1}^{N}\left\|\int_{t_{k-1}}^{t_{k}}\left(u^{\prime}(t)-\frac{u^{\prime}\left(t_{k}\right)+u^{\prime}\left(t_{k-1}\right)}{2}\right) d t\right\|_{V^{\prime}}^{2}+\tau^{2}\left(\left\|Q_{h}(0) u^{0}-u_{0}^{h}\right\|_{V}^{2}\right. \\
& +\max _{0 \leqslant t \leqslant T}\left\|\left(I-Q_{h}\right) u(t)\right\|_{V}^{2}+\int_{0}^{T}\left\|\left[I-Q_{h}(t)\right] u^{\prime}(t)\right\|^{2} d t \\
& \left.\left.+\frac{1}{\tau} \sum_{k=1}^{N}\left\|\int_{t_{k-1}}^{t_{k}}\left(\frac{u^{\prime}\left(t_{k}\right)+u^{\prime}\left(t_{k-1}\right)}{2}-u^{\prime}(t)\right) d t\right\|^{2}\right)\right\} .
\end{aligned}
$$


Из (3.12), учитывая представление

$$
\sum_{k=1}^{N}\left\|\left(z_{k}-z_{k-1}\right) \tau^{-1}\right\|^{2} \tau=\sum_{k=1}^{N}\left\|\frac{1}{\tau} \int_{t_{k-1}}^{t_{k}} u^{\prime}(t) d t-\frac{u_{k}^{h}-u_{k-1}^{h}}{\tau}\right\|^{2} \tau
$$

непрерьвность вложения $H \subset V^{\prime}$, ограниченность параметра $\tau$ и оценку

$$
\left\|Q_{h}(0) u^{0}-u_{0}^{h}\right\|_{V}^{2} \leqslant 2\left\|Q_{h} u^{0}-u_{0}^{h}\right\|_{V}^{2}+2 \max _{0 \leqslant t \leqslant T}\left\|\left[I-Q_{h}(t)\right] u(t)\right\|_{V}^{2},
$$

получим оценку (3.5).

Воспользуемся оценками выражения (2.13) из (3.3) и (3.4), которыеподставим в оценку (3.5). Получим

СлЕДСТВИЕ 2. Пусть выполнены условия гладкой разрешимости задачи (1.4), условие (2.2), и и(t) - решение задачи (1.4) такое, что $u^{\prime \prime} \in L_{p}(0, T ; H)$ для $1 \leqslant p \leqslant 2$, т.е. выполняется оченка (2.3). Пусть, кроме того, операторы $B(t)$ удовлетворяют условию (2.6). Тогда справедлива оченка

$$
\begin{aligned}
\max _{1 \leqslant k \leqslant N}\left\|u\left(t_{k}\right)-u_{k}^{h}\right\|^{2} \leqslant M & \left\{\left\|Q_{h} u^{0}-u_{0}^{h}\right\|_{V}^{2}+\left(\tau^{4}+h^{3}\right)\left(\max _{0 \leqslant t \leqslant T}\|A(t) u(t)\|^{2}\right.\right. \\
& \left.\left.+\int_{0}^{T}\left\|u^{\prime}(t)\right\|_{V}^{2} d t\right)+\tau^{3-2 / p}\left(\int_{0}^{T}\left\|u^{\prime \prime}(t)\right\|^{p} d t\right)^{2 / p}\right\} .
\end{aligned}
$$

Если же решение $u(t)$ такое, что $u^{\prime \prime \prime} \in L_{p}(0, T ; H)$ для $1 \leqslant p \leqslant 2$, т.е. выполняется оценка (2.4), то справедлива следующая оченка:

$$
\begin{aligned}
\max _{1 \leqslant k \leqslant N}\left\|u\left(t_{k}\right)-u_{k}^{h}\right\|^{2} \leqslant M & \left\{\left\|Q_{h} u^{0}-u_{0}^{h}\right\|_{V}^{2}+\left(\tau^{4}+h^{3}\right)\left(\max _{0 \leqslant t \leqslant T}\|A(t) u(t)\|^{2}\right.\right. \\
& \left.\left.+\int_{0}^{T}\left\|u^{\prime}(t)\right\|_{V}^{2} d t\right)+\tau^{5-2 / p}\left(\int_{0}^{T}\left\|u^{\prime \prime \prime}(t)\right\|^{p} d t\right)^{2 / p}\right\} .
\end{aligned}
$$

ДокАЗАТЕЛЬСТво. Для получения оценки (3.13) обратим внимание на очевидную оценку

$$
\max _{0 \leqslant t \leqslant T}\left\|\left(I-Q_{h}\right) u(t)\right\|_{V}^{2} \leqslant c h^{2} \max _{0 \leqslant t \leqslant T}\|A(t) u(t)\|_{H}^{2},
$$

а также воспользуемся оценкой, установленной в [2],

$$
\frac{1}{\tau} \sum_{k=1}^{N}\left\|\int_{t_{k-1}}^{t_{k}}\left(\frac{u^{\prime}\left(t_{k}\right)+u^{\prime}\left(t_{k-1}\right)}{2}-u^{\prime}(t)\right) d t\right\|^{2} \leqslant \frac{\tau^{3-2 / p}}{4}\left(\int_{0}^{T}\left\|u^{\prime \prime}(t)\right\|^{p} d t\right)^{2 / p} .
$$

Подставляя в правую часть (3.5) оценки $(2.16),(2.17),(3.15),(3.16)$ и (3.3), получим

$$
\begin{aligned}
& \max _{1 \leqslant k \leqslant N}\left\|u\left(t_{k}\right)-u_{k}^{h}\right\|^{2} \leqslant M\left\{\left\|Q_{h} u^{0}-u_{0}^{h}\right\|_{V}^{2}+h^{4}\left\|A(0) u^{0}\right\|^{2}\right. \\
& \quad+\tau^{3-2 / p}\left(\int_{0}^{T}\left\|u^{\prime \prime}(t)\right\|^{p} d t\right)^{2 / p}+\left[\left\|Q_{h} u^{0}-u_{0}^{h}\right\|_{V}+h\left(\max _{0 \leqslant t \leqslant T}\|A(t) u(t)\|\right.\right. \\
& \left.\left.\quad+\left(\int_{0}^{T}\left\|u^{\prime}(t)\right\|_{V}^{2} d t\right)^{1 / 2}\right)+\tau^{3 / 2-1 / p}\left(\int_{0}^{T}\left\|u^{\prime \prime}(t)\right\|^{p} d t\right)^{1 / p}\right] h^{2} \max _{0 \leqslant t \leqslant T}\|A(t) u(t)\| \\
& \left.\quad+\tau^{2}\left[\left\|Q_{h} u^{0}-u_{0}^{h}\right\|_{V}^{2}+h^{2}\left(\max _{0 \leqslant t \leqslant T}\|A(t) u(t)\|^{2}+\int_{0}^{T}\left\|u^{\prime}(t)\right\|_{V}^{2} d t\right)\right]\right\} .
\end{aligned}
$$


Оценка (3.13) получается теперь из (3.17) в результате простых преобразований.

Оценка (3.14) получается аналогично. Следует только вместо оценки (3.3) воспользоваться оценкой (3.4), а вместо оценки (3.16) применить оценку

$$
\frac{1}{\tau} \sum_{k=1}^{N}\left\|\int_{t_{k-1}}^{t_{k}}\left(\frac{u^{\prime}\left(t_{k}\right)+u^{\prime}\left(t_{k-1}\right)}{2}-u^{\prime}(t)\right) d t\right\|^{2} \leqslant \frac{\tau^{5-2 / p}}{64}\left(\int_{0}^{T}\left\|u^{\prime \prime \prime}(t)\right\|^{p} d t\right)^{2 / p}
$$

установленную также в [2].

ЗАмЕчАниЕ 2. Полученные выше результаты остаются верными, если равномерную сетку по времени заменить произвольной $0=t_{0}<t_{1}<\cdots<t_{N}=T$, удовлетворяющей условию $N \max \tau_{k} \leqslant C$, где $\tau_{k}=t_{k}-t_{k-1}$ и $k=\overline{1, N}$. При этом в соответствуюших оценках погрешности, например, (3.13) и (3.14), в правой части оценок под $\tau$ нужно понимать $\max \tau_{k}$.

\section{СПИСОК ЦИТИРОВАННОЙ ЛИТЕРАТУРЫ}

[1] Смагин В. В. Оценки в сильных нормах погрешности проекционно-разностного метода приближенного решения абстрактного параболического уравнения // Матем. заметки. 1997. T. 62 . №6. C. $898-909$.

[2] Смагин В. В. Оценки в сильных нормах погрешности проекционно-разностного метода для параболических уравнений с модифицированной схемой Кранка-Николсон // Матем. заметки. 2003. Т. 74. №6. С. 913-923.

[3] Смагин В. В. Оценки скорости сходимости проекционного и проекционно-разностного методов для слабо разрешимых параболических уравнений // Матем. сб. 1997. Т. 188. № 3. C. $143-160$.

[4] Смагин В.В. Среднеквадратичные оценки погрешности проекционно-разностного метода для параболических уравнений // ЖВМиМФ. 2000. Т. 40. №6. С. 908-919.

[5] Сьярле $\Phi$. Метод конечных элементов для эллиптических задач. М.: Мир, 1980.

[6] Смагин В.В. Оценки погрешности полудискретных приближений по Галеркину для параболических уравнений с краевым условием типа Неймана // Изв. вузов. Сер. матем. 1996. № 3(406). С. $50-57$.

[7] Марчук Г.И., Агошков В.И. Введение в проекционно-сеточные методы. М.: Наука, 1981.

[8] Лионс ЖК.-Л., Мадженес Э. Неоднородные граничные задачи и их приложения. М.: Мир, 1971.

[9] Смагин В.В. О разрешимости абстрактного параболического уравнения с оператором, область определения которого зависит от времени // Дифференц. уравнения. 1996. Т. 32. № 5 . C. 711-712.

[10] Вайникко Г. М., Оя П. Э. О сходимости и быстроте сходимости метода Галеркина для абстрактных эволюционных уравнений // Дифференц. уравнения. 1975. Т. 11. № 7. С. 1269-1277. 